\section{Erste Schritte in die Program- morientierte Förderung - Ein Abenteuerbericht}

\author{
von Manfred Popp, Forschungszentrum \\ Karlsruhe
}

Im September 2001 wurde für die Hermann von Helmholtz-Gemeinschaft Deutscher Forschungszentren (HGF), die die 15 Großforschungseinrichtungen in Deutschland umfasst, das neue Instrument der „Programmorientierten Förderung" eingeführt. Nach der Evaluation von zwei der sechs Forschungsbereiche der HGF zeigt der Bericht die im Verlauf der Verfahren gewonnenen positiven Ergebnisse wie auch negative Erfahrungen und Sorgen in Hinblick auf die zukünftige Entwicklung auf.

Nach Abschluss der 90er Jahre, die vielleicht als Jahrzehnt der Evaluation, wenn nicht der „Evaluitis" in die deutsche Wissenschaftsgeschichte eingehen werden, hat die Helmholtz-Gemeinschaft mit der im September 2001 eingeführten Programmorientierten Förderung das System der Wissenschaftssteuerung durch Evaluierung $\mathrm{zu}$ einer neuen, bisher einzigartigen Form und Dimension weiterentwickelt. Ausgangspunkt war die Einsicht, dass die Fortsetzung der reinen institutionellen Förderung von 15 Großforschungseinrichtungen, auch wenn sie sich in der 1995 gegründeten Helmholtz-Gemeinschaft verstärkt aufeinander abstimmten und $\mathrm{zu}$ gemeinsamen Programmen fanden, auf die Dauer nicht aufrechtzuerhalten wäre. Die Bewilligung von Programmen der Zentren, die zusammen genommen die größte Wissenschaftsorganisation Deutschlands darstellen, ohne vorherige Begutachtung und nur mit ex post-Evaluierungen und Bewertungen durch wissenschaftliche Beiräte, erschien vielen im deutschen Wissenschaftssystem als nicht ausreichender Garant für wissenschaftliche Qualität und richtige strategische Ausrichtung der Programme. Auch war klar, dass die Helmholtz-Zentren sich künftig verstärkt einem Wettbewerb untereinander stellen müssen. Dazu war bereits Ende der 90er Jahre das Instrument des Strategiefonds geschaffen worden, das zwar nur $5 \%$ der insgesamt für die HGF verfügbaren Mittel durch ein wettbewerbliches Verfahren um Finanzierung von dazu eigens beantragten Projekten verteilte, jedoch außerordentlich viele Anregungen in den
Helmholtz-Zentren gebracht, viele Kooperationen befördert und viele Umorientierungen in den Zentren eingeleitet hat. Leider ist dieses intelligente Instrument der inkrementalen Steuerung wissenschaftlicher Kapazitäten nie wirklich einmal gesondert bewertet und als solches evaluiert worden, sondern nach dem Wechsel der Bundesregierung im Jahr 1998 zu Gunsten der Programmorientierten Förderung ganz aufgegeben worden.

Grundgedanke der Programmorientierten Förderung ist die strategische Bewertung der gesamten Forschungsprogramme der Helmholtz-Zentren in sechs Forschungsbereichen, also die hundertprozentige Erfassung der Helmholtz-Programme. Die Zentren bewerben sich allein oder in Kooperation untereinander um Anteile an den Programmen. Entsprechend dem Ergebnis der Begutachtung erhalten sie nach Befassung des Senats die entsprechenden Finanzierungsanteile in ihre Haushalte eingestellt.

Nach der ersten Runde der Begutachtung für die Forschungsbereiche „Gesundheit" und „Verkehr und Weltraum“ muss man zunächst erleichtert feststellen, dass das völlig neuartige Verfahren einer strategischen Begutachtung sich tatsächlich als praktikabel erwiesen hat.

Die einzelnen Programme der HelmholtzGemeinschaft wurden auf 30 Seiten plus 50 Seiten Anhang dargestellt und umfassten in der Regel ein Volumen von mindestens dem 10 fachen eines Sonderforschungsbereichs der Deutschen Forschungsgemeinschaft. Dies erforderte von den darstellenden Zentren, aber erst recht von den Gutachtern eine erhebliche Bereitschaft zu Abstraktion, zu Konzentration auf die wesentlichen Kernaussagen und die Bereitschaft der Gutachter, auf dieser Basis erhebliche Haushaltsmittel letzten Endes quer zu schreiben. Dies ist zu meiner Überraschung in den meisten Fällen gelungen. Wo sich Gutachterausschüsse dann doch auf die Behandlung einzelner Projekte im Detail eingelassen haben, war dies stets durch eine entsprechende Darstellung in den Programmen provoziert und damit von dem jeweiligen Helmholtz-Zentrum selbst verschuldet. Die anschließende Beratung und Zusammenführung der einzelnen Gutachtervoten im Senatsausschuss bzw. im Senat hat insgesamt zu deutlich sichtbaren Verschiebungen der Prioritäten innerhalb des Gesundheitsprogramms geführt. 


\section{Reform der Helmholtz-Gemeinschaft - Programmorientierte Förderung und Wissenschaftsadäquates Controlling}

Mit der Gründung der Helmholtz-Gemeinschaft e.V. hat die strategische Neuorientierung der HelmholtzGemeinschaft, früher Arbeitsgemeinschaft der Großforschungseinrichtungen, begonnen. Mit einem neu entwikkelten Konzept der Programmorientierung und programmorientierten Förderung wird von der bisherigen zentrenorientierten Steuerung zu einer thematisch orientierten Steuerung und Förderung übergegangen. Die HelmholtzZentren haben sich daher im September 2001 in Form eines eingetragenen Vereins zusammengeschlossen. Die Organe des Helmholtz-Vereins sind (vgl. nebenstehende Grafik, in der die Struktur dargestellt ist und beispielhaft die Aufgaben des Senats beschrieben sind):

- der Senat, mit Mitgliedern aus Wissenschaft, Wirtschaft und Politik,

- der Ausschuss der Zuwendungsgeber,

- die Mitgliederversammlung, repräsentiert von den Vorständen der Helmholtz-Zentren,

- der zentrenneutrale hauptamtliche Präsident.

Die drei tragenden Säulen des neuartigen, auf Kooperation und Wettbewerb ausgelegten Fördersystems sind die programmorientierte Förderung, das wissenschaftsadäquate Controlling sowie die Einführung von Flexibilisierungsinstrumenten im Personalbereich (Finanzquote für unbefristete Stellen anstelle der bisherigen starren Stellenpläne) und in der Haushaltsführung (überjährige Mittelbewirtschaftung).

\section{Programmförderung anstelle der Institutionenförderung}

Bis zum Jahr 2002 erhielten die Helmholtz-Zentren individuelle Jahresbudgets durch die Zuwendungsgeber. Mit Einführung der programmorientierten Förderung ab dem Jahr 2003 werden die Mittel künftig auf der Grundlage von längerfristig angelegten Forschungsprogrammen verteilt. Im Rahmen von insgesamt sechs thematisch orientierten HGF-Forschungsbereichen erarbeiten die Wissenschaftler in den Helmholtz-Zentren und in Kooperation über die Zentrengrenzen hinweg eigenverantwortlich Programmbeiträge, mit denen sie sich dem Wettbewerb um Ressourcen stellen. In einem abgestuften Evaluationsverfahren bewertet der Helmholtz-Senat mit Hilfe externer Experten aus dem In- und Ausland diese Programme und formuliert Förderempfehlungen an die Zuwendungsgeber. Auf der Grundlage dieser Empfehlungen erhalten die Helmholtz-Zentren für ihre positiv bewerteten Programmbeiträge die notwendigen Ressourcen. Der Präsident, unterstützt von der Helmholtz-Geschäftsstelle, managt den Evaluationsprozess, bereitet die Entscheidungen des Senats vor und setzt sie um. Das Gesamtbudget eines Forschungszentrums setzt sich schließlich aus der Summe seiner Programmbeiträge zu den sechs HGFForschungsbereichen zusammen. Die Begutachtungen werden im Abstand von fünf Jahren durchgeführt, so dass die Förderentscheidungen jeweils für eine 5-Jahres-Periode Wirkung haben. In einem zeitversetzten Verfahren werden jeweils zwei HGF-Forschungsbereiche in einem Jahr evaluiert.

\section{Begutachtung der Programme und Finanzierungskonsequenzen}

Im Sinne von Globalsteuerung formulieren die Zuwendungsgeber im Zusammenwirken mit den Helmholtz-Zentren forschungspolitische Vorgaben für die Programme der sechs HGF-Forschungsbereiche: Energie, Erde und Umwelt, Gesundheit, Schlüsseltechnologien, Struktur der Materie, Verkehr und Raumfahrt: die Forschungsbereiche sind in Programme und Programmthemen weiter untergliedert. Die forschungspolitischen Vorgaben umreißen Aufgabenschwerpunkte und den Finanzkorridor für die jeweilige Planperiode. Vor diesem Hintergrund finden die Begutachtungsverfahren statt, die am Ende in konkrete Finanzierungsentscheidungen münden. Auf der Grundlage von strategisch ausgerichteten Programmproposals und einer zweitägigen Gutachtersitzung mit Diskussion der beteiligten Wissenschaftler wird eine inhaltlich begründete und benotete Gutachterempfehlung abgegeben. Zentrale Kriterien sind die wissenschaftliche Qualität, die strategische Relevanz und die effiziente Durchführung. Die Gesamtheit der begutachteten Programme wird schließlich im Senatsausschuss für die Begutachtung weiter beraten und mit einer Förderempfehlung in den Senat eingebracht. Der Helmholtz-Senat schließlich gibt eine abschließende Förderempfehlung zur Durchführung der positiv bewerteten Programme ab. Auf Ebene des Ausschusses der Zuwendungsgeber wird diese Förderempfehlung schließlich in eine Finanzierungsentscheidung für die Programme und die beteiligten Helmholtz-Zentren für die Begutachtungsperiode von fünf Jahren umgesetzt. Das Ergebnis fließt dann programmbezogen in die jährlichen Wirtschaftspläne der Helmholtz-Zentren ein. 


\section{Wissenschaftsadäquates Controlling}

Als Element des wissenschaftsadäquaten Controllings enthalten die auf die Zukunft ausgerichteten, strategischen Programmproposals auch über 5-Jahres-Zeiträume rückwärts gerichtete Erfolgsdarstellungen. Dabei wird die inhaltliche Beschreibung erzielter Forschungserfolge durch wissenschaftsimmanente Kenngrößen, wie z.B. referierte Publikationen, C-Rufe, Patente oder Lizenzeinnahmen ergänzt. Die Abwicklung der begutachteten Programme wird von einem jährlichen Controlling in Form eines standardisierten, inhaltlichen und zahlenmäßigen Berichtswesen begleitet, um den Bedarf für notwendige Modifikationen oder sogar Richtungsänderungen in der Programmabwicklung besser erkennen zu können.

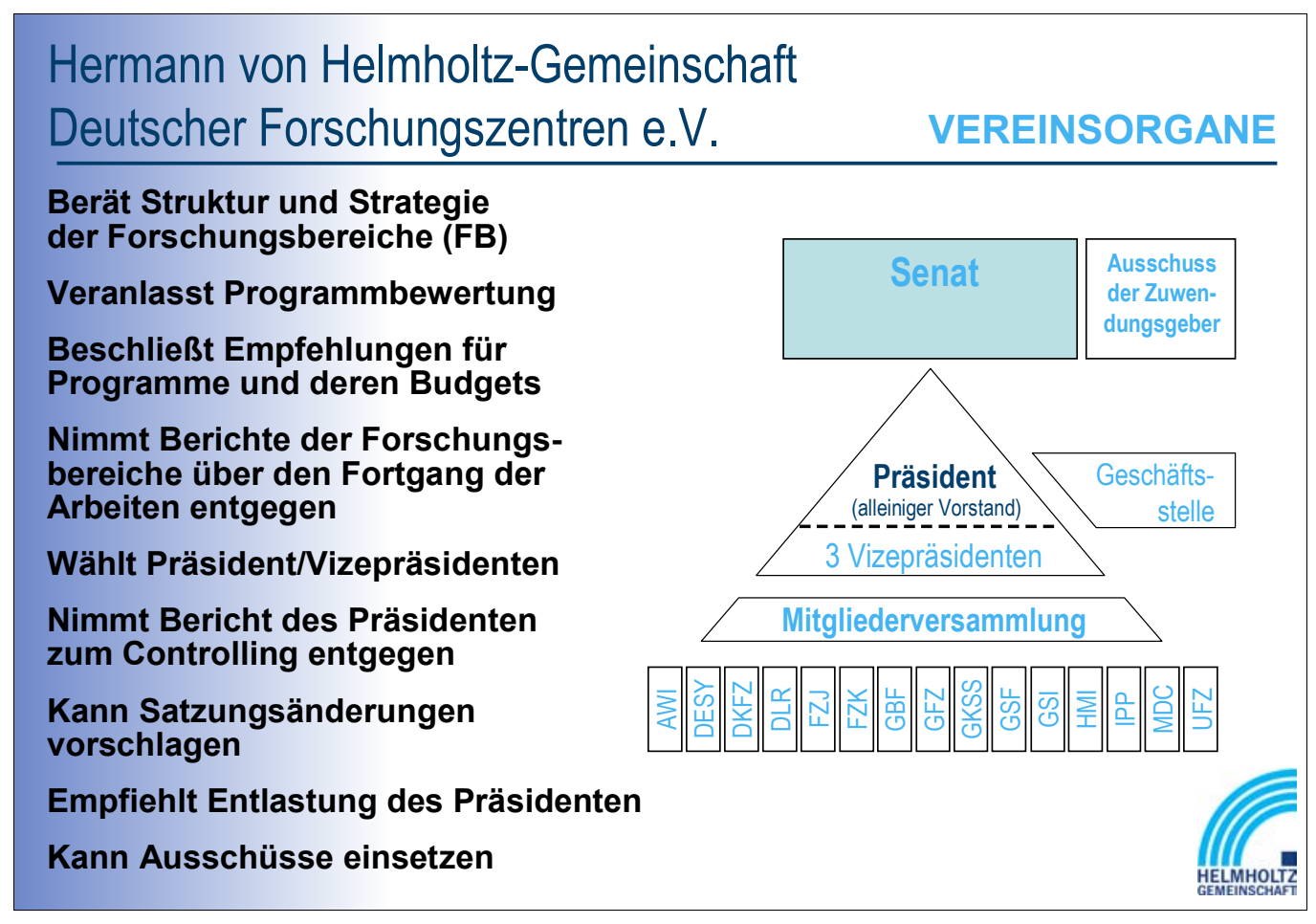

AWI Stiftung Alfred-Wegener-Institut für Polar- und Meeresforschung

DESY Stiftung Deutsches Elektronen-Synchrotron

DKFZ Stiftung Deutsches Krebsforschungszentrum

DLR Deutsches Zentrum für Luft- und Raumfahrt e.V.

FZJ Forschungszentrum Jülich $\mathrm{GmbH}$

FZK Forschungszentrum Karlsruhe GmbH

GBF Gesellschaft für Biotechnologische Forschung mbH

GFZ Stiftung GeoForschungZentrum Potsdam

GKSS Forschungszentrum Geesthacht GmbH

GSF Forschungszentrum für Umwelt- und Gesundheit $\mathrm{GmbH}$

GSI Gesellschaft für Schwerionenforschung mbH

HMI Hahn-Meitner-Institut Berlin GmbH

IPP Max-Planck-Institut für Plasmaphysik

MDC Stiftung Max-Delbrück-Centrum für Molekulare Medizin

UFZ Umweltforschungszentrum Leipzig GmbH 
Damit ist auch der Nachweis erbracht, dass forschungspolitische Weichenstellungen auf diesem Wege möglich sind.

Als zweites positives Ergebnis ist festzuhalten, dass die Gutachter der Arbeit in den Helmholtz-Zentren erstklassige Noten gegeben haben. Sowohl im Bereich der Verkehrs- und Weltraumforschung wie in der Gesundheitsforschung wurde den Arbeiten bescheinigt, dass sie international auf höchstem Standard und in vielen Bereichen führend sind. Damit ist die jahrzehntelange vielstimmige Kritik an den Großforschungseinrichtungen nun nachprüfbar als Vorurteil entlarvt. Überspitzt könnte man sagen, dass der Reformbedarf in den Großforschungseinrichtungen doch nicht so groß war, wie immer behauptet. In jedem Fall ist aber die Sichtbarmachung dieser Qualität ein großer Vorteil der Programmorientierten Förderung auch für die Helmholtz-Zentren.

Aber es gibt natürlich auch negative Erfahrungen und Sorgen nach der ersten Runde der Programmorientierten Förderung. Eine ernste Sorge betrifft die Zerlegung der HelmholtzGemeinschaft in ihre einzelnen Programme. Diese Gliederung in Programme in den einzelnen Forschungsbereichen ist notwendig, um eine für die Begutachtung geeignete Strukturierung zu erzeugen, sie ist fachlich nicht immer zwingend. So hätte sich zum Beispiel der Forschungsbereich „Gesundheit" nicht unbedingt in sieben Programme untergliedern müssen; $\mathrm{Zu}$ schnitt und Unterteilung der Programme waren ein langer Diskussionsprozess, der durchaus auch zu anderen Lösungen hätte führen können. Angesichts dieser relativen Bedeutung der Programmgrenzen ist die ausschließliche Konzentration der Gutachter auf das ihnen vorgelegte Programm besorgniserregend, weil auf diese Weise wichtige Verbindungen der Programme untereinander oder der Programme zu anderen Stärken eines Zentrums unsichtbar bleiben. Gerade die wechselseitige Befruchtung unterschiedlicher Programme in einem Zentrum und die Vernetzung und Bündelung vieler Vorhaben ist aber ein Markenzeichen der HelmholtzGemeinschaft und eine für die Bearbeitung komplexer Wissenschaftsthemen notwendige Organisationsform. Eine zu starke Fokussierung der Begutachtung auf einzelne Programme würde deshalb gerade einer der besonderen Spezifika der Helmholtz-Forschung zuwiderlaufen. Es wäre auch wenig gewonnen, würde man die bisher isolierte Betrachtung von 15 Zentren durch eine künftig isolierte Betrachtung von ca. 25 Programmen ersetzen. Hier muss also dringend für eine bessere Querverbindung zwischen den Programmen und eine bessere Sichtbarkeit der übergeordneten Verbindungen im Begutachtungsprozess gesorgt werden.

Eine zweite Sorge betrifft die mit der Programmorientierten Förderung verbundene Fixierung der Programme, die nach Begutachtung ja weitgehend für fünf Jahre festgelegt sind. Zwar sind die Helmholtz-Zentren mit $20 \%$ ihres Budgets programmungebunden; sie sind damit in der Lage, neue Themen aufzugreifen oder wichtige Korrekturen vorzunehmen. Andererseits wird aber erwartet, dass das Ergebnis der Begutachtungen auch nachvollziehbar in Programmen umgesetzt und über das Controlling nachverfolgt wird. Hier besteht vor allem die Sorge, dass notwendige Abweichungen von einmal vorgesehenen Programmen mit dem Fortschritt der Wissenschaften, der sicherlich noch Gutachterausschüssen verständlich gemacht werden kann, bei der Weiterverfolgung durch Bürokratie oder Rechnungshöfe zu einer unangemessenen Fesselung der Dynamik von Entwicklungen im wissenschaftlichen Bereich führen könnte.

Nicht ohne Sorge blickt man auf den ungeheueren Arbeitsaufwand zurück, der für die Begutachtung in einem Forschungsbereich notwendig war und der für die ausstehenden Begutachtungen in den anderen vier Forschungsbereichen noch geleistet werden muss. Hier werden erhebliche Ressourcen gebunden, oft gerade auch Zeit und Kapazität der besten Wissenschaftler. In vielen Programmen des Forschungsbereichs „Gesundheit" steht die Beeinflussung der Weiterentwicklung der Programme durch den Begutachtungsprozess in keinem günstigen Verhältnis zu dem betriebenen Aufwand. Es wäre deshalb sehr wichtig gewesen, wenn die versprochene Belohnung für die Einführung der Programmorientierten Förderung und die damit gezeigte Reformbereitschaft durch entsprechende Zuwachsraten im Haushalt der Helmholtz-Gemeinschaft auch eingetreten wäre und damit nach langen Jahren der Stagnation wieder ein echter Zuwachs in den Forschungsprogrammen der Helmholtz-Zentren möglich geworden wäre. Leider hat die Ent 
wicklung der öffentlichen Haushalte ab dem Jahr 2003 einen kräftigen roten Strich durch diese Erwartungen gemacht, was die Akzeptanz der Programmorientierten Förderung bei den Wissenschaftlern sicherlich nicht gefördert hat.

Es ist also ein gemischtes Bild, das sich nach den ersten Schritten in die Programmorientierte Förderung bietet und es ist aus meiner Sicht noch nicht eindeutig, ob sich die Programmorientierte Förderung als Segen für die Helmholtz-Gemeinschaft erweist. Es lauern viele Gefahren an dem weiteren $\mathrm{Weg}$ - die $\mathrm{Zu}-$ sammenballung der Helmholtz-Gemeinschaft in einem Konzern, eine zu starke bürokratische Kontrolle der bewilligten Forschungsprogramme, ein zu großer Aufwand für die Durchführung der Programmorientierten Förderung und eine neue Form der Versäulung in zu stark separat betrachteten Programmen. Es wird also sehr darauf ankommen, dass die Reformbereitschaft mit der Einführung der Programmorientierten Förderung, so wie sie im Jahr 2001 konzipiert wurde, nicht zu Ende ist, sondern auch die Bereitschaft bei allen Beteiligten da ist, aus den sich abzeichnenden Erfahrungen die richtigen Konsequenzen zu ziehen und die Instrumente der Programmorientierten Förderung intelligent weiterzuentwickeln.

\section{Kontakt}

Prof. Dr. Manfred Popp

Vorstandsvorsitzender des Forschungszentrums Karlsruhe $\mathrm{GmbH}$

Postfach 36 40, 76021 Karlsruhe

E-Mail: manfred.popp@vorstand.fzk.de

Internet: http://www.fzk.de

\section{Kontrolle ist gut, Vertrauen ist besser Zur Entwicklungsgeschichte von
Steuerung und Erfolgskontrolle in
der Helmholtz-Gemeinschaft}

von Henning Möller, Forschungszentrum Karlsruhe

Steuerung und Erfolgskontrolle der staatlich finanzierten, rechtlich unabhängigen Großforschungszentren, heute HelmholtzZentren, waren schon in der Gründungsphase von Bedeutung. Verschiedene Verfahren der Erfolgskontrolle wurden dem jeweiligen Zeitgeist entsprechend angewandt. So lässt sich eine Entwicklungsgeschichte vom Nachweis der wirtschaftlichen Verwendung der staatlichen Zuwendungen für die Großforschungszentren bis hin zum wissenschaftsadäquaten Controlling der neuerdings eingeführten programmorientierten Förderung der Helmholtz-Gemeinschaft (HGF) verfolgen. Aus dem Erfolg der frühen Großforschung lässt sich die These ableiten, dass eine standardisierte Erfolgskontrolle nur ein Baustein für die Steuerung von Forschung sein kann, vorrangig bedarf es aber eines Grundvertrauens und Aufgabenkonsenses zwischen Zuwendungsgebern, Gesellschaft, Adressaten der Forschung und forschenden Zentren, wenn Erfolgskontrolle nicht zum formalisierten Disziplinierungsinstrument degradiert werden soll. In der typischerweise mittel- und langfristig angelegten Forschung der HelmholtzZentren, der Orientierung auf Vorsorgeaufgaben und dem Betrieb komplexer Großgeräte lässt sich Erfolg kaum in der industriegängigen Kennziffer ,jährlicher Gewinn“ messen. Die Verknüpfung von Erfolgsfaktoren ex post mit Programmstrategien ex ante kann bei richtiger Anwendung zu einem geeigneten Steuerungsinstrument werden. Dagegen zeigen negative Erfahrungen, dass beim Einsatz von Begutachtungsverfahren mit starren Finanzierungskonsequenzen der Anpassungsdruck an Erfolgskennziffern zum dominierenden Faktor der Forschungsleistung werden kann. 